\title{
Kollokationsforschung und Kollokationsdidaktik
}

\author{
Anna Reder (Pécs)
}

\begin{abstract}
Investigating and teaching collocations. The starting point of the paper is the observation that foreign language learners translate collocations literally from their L1. Collocations are conceived here as phrasal units which can be placed between free word groups and idioms. The paper focuses on metaphoric collocations, i.e. with polysemic collocator. In this type of collocation the collocator is used in a metaphoric meaning, while it appears in denotative meaning in free word groups. Contrasting types of collocation are presented in a model. Data from empirical research show that foreign language learners of a certain level regard metaphoric collocations as marked and transfer them less often than non-metaphoric collocations. The paper supports the need for pointing out the significance of collocations even for beginners.
\end{abstract}

\section{$1 \quad$ Kollokationsfehler}

Der folgende Dialog, der sich zwischen einem Studierenden und einem Lehrenden an der Universität Pécs/Ungarn zu Beginn eines Semesters in der Anmeldungswoche abspielte, liefert einen exemplarischen Beleg dafür, dass Kollokationen auch für fortgeschrittene Lerner große Schwierigkeiten bereiten können:

Student: Ich möchte Ihre Lehrveranstaltung <aufnehmen>.

Lehrender: Bringen Sie bitte unbedingt einen Kassettenrecorder mit.

Dieses kurze Gespräch belegt jeweils eine Strategie der Akteure. Der Lerner stützt sich auf seine Erstsprache Ungarisch und nimmt einen Bedeutungstransfer vor. Der Kollokator aufnehmen ist nämlich die wörtliche Übersetzung des Verbs felvesz, das im Ungarischen im Kontext von Lehrveranstaltung metaphorisch in der Bedetung von 'belegen' verwendet wird. Diese übertragene Bedetung hat jedoch dt. aufnehmen in diesem Kontext nicht.

Wie kann der Lehrende auf diesen Satz reagieren? Im obigen Beispiel bedient er sich der Strategie der humorvollen Fehlerandeutung, indem er scherzhaft darauf verweist, dass der Student einen Kollokationsfehler begangen hat. Er bringt eine andere Lesart des Verbs aufnehmen ins Spiel, nämlich 'aufzeichnen', und somit den Studenten ins Grübeln.

Mit solchen Kollokationsfehlern kann der Lehrende jedoch unterschiedlich umgehen. Er hat auch die Möglichkeit, weitgehende Fehlertoleranz auszuüben. Denn der situative Kontext macht das Anliegen des Studierenden so eindeutig, dass der Lehrende den Satz auch mit dem im Deutschen unüblichen Kollokator verstehen kann. In diesem Fall stört der Kollokationsfehler die Kommunikation nicht wirklich. Wollte man jedoch den Lerner schon in der Anmel- 
dungswoche auf die Nichtäquivalenz der Kollokationsbestandteile im Deutschen und Ungarischen schon aufmerksam machen, wäre es ratsam, eine direkte oder indirekte Korrektur durchzuführen. Die Kollokationsgleichung

(1) einen Kurs belegen $\approx$ egy kurzust felvesz

ist eine unter zahlreichen, in denen sich die Kollokatoren in Zielsprache und Ausgangssprache unterschiedlicher Metaphorik bedienen und dadurch Lernschwierigkeiten verursachen können. Aus diesem Grunde stehen die metaphorisch gebrauchten Kollokationen sowie jene Fehler, die bei deren Verwendung durch Fremdsprachenlerner begangen werden im Mittelpunkt des vorliegenden Aufsatzes. Als Einstieg in die Thematik dienen im folgenden Kapitel einige Überlegungen zum Terminus Kollokation.

\section{$2 \quad$ Kollokation - ein Modewort}

Im Zeitalter der Informationsgesellschaft und der hochentwickelten Computerwelt gibt es in der Linguistik viele neue Bezeichnungen und auch einen etwas älteren Terminus, Kollokation, der jedoch Konjunktur hat. Er begegnet uns auf Schritt und Tritt bzw. Klick, denn mehrere elektronische Korpora bieten unter anderem auch Kollokationsrecherchen an. Obwohl das Wort Kollokation ein linguistischer Terminus ist und häufig in der einschlägigen Literatur Anwendung findet, lässt sich kein einheitliches Kollokationsverständnis feststellen. Der Terminus ist polysem. In verschiedenen Disziplinen der Linguistik gibt es verschiedene Deutungen, die sich zum Teil noch in Entwicklung befinden. Die eher klassische Auffassung ist jedoch die kontextualisitische, auf die im Folgenden kurz eingegangen wird.

\subsection{Kollokation - statistische Häufigkeit des Miteinandervorkommens binärer lexi- kalischer Einheiten}

Im korpuslinguistischen Zugang wird Kollokation in der Regel als die statistische Häufigkeit des Miteinandervorkommens von binären lexikalischen Einheiten in vordefinierten Texten gedeutet. Im Kontextualismus und - in Anlehnung daran - in der Korpuslinguistik steht also das Wort in konkreten Texten im Mittelpunkt und bildet Kollokationen mit anderen Wörtern, mit denen es häufig vorkommt. Das Computerzeitalter fördert diese Kollokationsauffassung durch seine technischen Umsetzungsmöglichkeiten. Mit der Steigerung der Speicherkapazität hat man Zugriff auf eine große Menge von Texten. Es ist heute möglich, in außerordentlich umfangreichenTextkorpora ${ }^{1}$ das kollokative Verhalten eines Wortes zu untersuchen. Wenn man jedoch versucht, in einem Korpus die Kollokationen eines Wortes maschinell zu suchen, bekommt man häufig sehr überraschende Ergebnisse. Zum einen wird man je nach Korpus mit unterschiedlichen Kollokationen konfrontiert und zum anderen zeigen die Kookkurrenzlisten, dass der Computer oder genauer gesagt die Software die Polysemie der Wörter nicht handhaben kann.

\footnotetext{
1 Textkorpora, in denen man nach Kollokationen recherchieren kann, gibt es für das Deutsche z. B. unter der folgenden Website: <http://www.ids-mannheim.de/cosmas>. Für das Ungarische ist das "Magyar Nemzeti Szövegtár" unter <http://corpus.nytud.hu> sowie das "Magyar Történeti Korpusz" unter $<$ http:www.nytud.hu/hhc $>$ zu finden.
} 


\subsection{Kollokation - semantische Verträglichkeit}

Die maschinell erstellten Kollokationslisten können natürlich der Polysemie der Wörter kaum Rechnung tragen, werfen jedoch eine große Menge von Kookkurrenzen in einem Text aus, die manuell noch vielseitig ausgewertet werden kann. Aus einer semantisch geprägten Perspektive kann jedoch unter Kollokation oder kollokable Bedeutung eine syntagmatische Relation zwischen miteinander vorkommenden Wörtern verstanden werden; diese Verwendung der genannten Termini ist in etwa synonym mit Verträglichkeit oder Kompatibilität. Vergleicht man jedoch die Verträglichkeit der Wörter in zwei Sprachen, stößt man häufig auf Wortverbindungen, die die Idiosynkrasie der Sprachen belegen, wie in den Beispielen (1) und (2):

(2) einen Film entwickeln $\approx$ filmet elóhív (= 'einen Film <hervorrufen>').

Dass man einen Film entwickeln kann, lässt sich durch die semantische Verträglichkeit zwischen Film und entwickeln in übertragener Bedeutung erklären. Die Kollokation (2) kann von der konkreten Bedeutung semantisch abgeleitet werden. Die konkrete Bedeutung von entwickeln ist nämlich "allmählich entstehen, sich stufenweise herausbilden" (Duden Universalwörterbuch 2003). So ein Prozess vollzieht sich wohl auch bei der Filmentwicklung.

Warum schließen aber z. B. einen Film und hervorrufen im Deutschen einander aus? Gibt es dafür eine semantische Erklärung? Besonders interessant ist die Frage für einen DaFLernenden aus der Perspektive seiner Erstsprache oder einer weiteren Fremdsprache, wenn eine Selektionsbeschränkung jeweils lediglich in der einen Sprache festgelegt ist, wie das in den Beispielen (1) und (2) der Fall ist.

Mit Hilfe des Begriffsinventars der strukturalistischen Semantik kann man bekanntlich den Versuch unternehmen, Kookkurrenzen sprachintern zu begründen. Die Verträglichkeit von Wörtern kann häufig mit Hilfe von Semen beschrieben werden. Die semantische Valenz operiert auch mit der Angabe von Bedeutungseinheiten. Ein plausibles Beispiel dafür ist das Verb denken, dessen syntaktische Valenz in der Lesart 'überlegen, geistig arbeiten' eine Nominativergänzung fordert. Die semantische Valenz wird mit Hilfe des Sems [Hum] (= menschliches Wesen) angeführt (Helbig/Schenkel 1991: 182). Diese Verbindbarkeitsregel würde natürlich einen unkonventionellen Umgang mit der Sprache, wie in etwa der Stein denkt, ausschließen. Es gibt jedoch zahlreiche Wörter, die sowohl in konkreter als auch in übertragener Bedeutung in Wortverbindungen auftreten können.

Als weiteres Beispiel soll das Verb besuchen herangezogen werden, um zu zeigen, wie sich dessen Verträglichkeit mit anderen Wörtern im Satz mit Hilfe der semantischen Valenz beschreiben lässt. Das Verb besuchen in konkreter Bedeutung fordert eine Person, z. B. die Oma besuchen. Eine weitere Lesart ergibt sich für das Verb besuchen im Beispiel (3), denn es fordert in übertragener Bedeutung ein Substantiv im Akkusativ, das das Sem 'Bildungseinrichtung (im weitesten Sinne)' beinhaltet, ${ }^{2}$ so z. B.

\footnotetext{
${ }^{2}$ Diese Bedeutungsbeziehung kann auch in der Terminologie von Coseriu als Selektion bezeichnet werden (vgl. Coseriu 1967: 296).
} 
die Schule besuchen.

Ergibt sich aber im Einzelfall immer eine semantische Erklärung dafür, dass bestimmte Wörter mit bestimmten anderen Wörtern in konkreten Texten gemeinsam auftreten? Gewiss nicht, denn die Semangabe ist für die Beschreibung der kollokablen Beziehung der Wörter z. B. für Idiome nicht ohne Einschränkung anwendbar. Bei dem Idiom etwas macht Schule kann man natürlich das Miteinandervorkommen der Wörter nicht dadurch erklären, dass man miteinander verträgliche, kompatible Bedeutungseinheiten der Bestandteile anführt. Man kann unter synchronem Aspekt nicht unbedingt semantisch erklären, warum Schule und machen zusammen vorkommen können. ${ }^{3}$ Das wird von Idiomen auch nicht erwartet. Es ist eben ihr Wesen, dass sie nicht kompositionell sind. Es gibt jedoch auch eine Vielzahl nichtidiomatischer Wortverbindungen, in denen sich die Verbindbarkeit der Bestandteile weniger durch kompatible Bedeutungsbestandteile erklären lässt, sondern eher auf Konvention beruht. Die Idiosynkrasie wird, wie schon oben angedeutet, aus der Perspektive einer weiteren Sprache leicht sichtbar. Eine weitere Kollokationsgleichung (4) soll dieses Phänomen in deutschungarischer Relation veranschaulichen:

(4) die vorliegende Arbeit $\approx$ a jelen tanulmány (=<anwesende $>$ Arbeit $)$.

Auch bei Nichtidiomen kann es also vorkommen, dass es für das Miteinadervorkommen der Wörter keine semantische Erklärung gibt. Genauer gesagt, wir können nicht unbedingt in jedem Einzelfall erklären, warum andere, eigentlich kompatible Wörter doch nicht gebräuchlich sind.

Zusammenfassend lässt sich feststellen, dass man Kollokation im Sinne von syntagmatische Verträglichkeitsbeziehung in der Praxis nicht immer durch die Angabe der kompatiblen Seme (Bedeutungseinheiten) belegen kann. Darüber hinaus ist zu beobachten, dass Wörter, die in einer Sprache miteinander kompatibel sind, in einer anderen Sprache wegen deren Selektionsbeschränkungen möglicherweise nicht verträglich sind.

\subsection{Kollokation - eine phraseologische Einheit}

Die traditionelle Kollokationsauffassung auf der Relationsebene, die durch die Verträglichkeitsbeziehung sehr weit gefasst ist, lässt sich auf der konkreten Ebene etwas einengen, indem Kollokation als eine wortübergreifende Entität des Wortschatzes gedeutet wird. Bezüglich der Kollokationsauffassung ist in der Phraseologieforschung jüngst die Tendenz zu beobachten, den Terminus Kollokation zur Bezeichnung fester nichtidiomatischer Wortverbindungen zu verwenden (vgl. Burger 1998: 38, Teubert 2004). Damit ist die Kollokation an der Schnittstelle zwischen freien Wortverbindungen und Idiomen angesiedelt. Von einer Schnittstelle kann man vielleicht gar nicht sprechen, eher von einer Zwischenposition, denn die Grenzen zwischen den einzelnen Einheiten sind eher fließend. Auch die Ansiedlung der Kollokationen in der Phraseologie kann jedoch das Problem nicht lösen, dass die metaphorische Lesart eines Kollokators häufig sprachspezifisch ist und auf Konvention beruht. Die Erfassung der Kollo-

\footnotetext{
${ }^{3}$ Für viele Idiome gibt es jedoch eine etymologische Erklärung, die eventuell auch das Miteinadervorkommen der Bestandteile erklärt.
} 
kation als Wortschatzeinheit könnte bei der Aneignung der Kollokationen durch Fremdsprachenlerner lernfördernd wirken, da eine Kollokation als eine wortübergreifende Einheit präsentiert, eingeübt und angewendet werden kann. Diese Annahme gibt Anlass dazu, sich aus fremdsprachendidaktischer Sicht der phraseologischen Kollokationsauffassung anzuschließen.

\section{Metaphorische vs. nichtmetaphorische Kollokationen}

Einige kompositionelle Wortverbindungen können, wie durch die Beispiele mit besuchen und entwickeln im Kapitel 2. (Beispiele 2, 3) veranschaulicht, Bestandteile enthalten, die je nach Kontext in konkreter oder in übertragener Bedeutung verwendet werden können. Analog zu den beiden Beispielen verhält es sich mit dem Verb versetzen. In konkreter Verwendung

(5) wird eine Mauer, ein Knopf versetzt

(5a) ein Beamter wird versetzt;

d. h. im Falle eines Gegenstandes, dass dieser 'an eine andere Stelle gebracht wird', bzw. im Falle eines Menschen, dass diesem ein anderer Arbeitsplatz zugewiesen wird. Hier wird das Verb in wörtlicher Bedeutung eingesetzt. In übertragener Verwendung des Kollokators ergibt sich im schulischen Kontext die folgende Kollokation:

(6) ein Schüler wird versetzt,

mit der Bedeutung, dass der Schüler 'an eine andere Stelle' nämlich in die nächste Klasse kommt. In der metaphorischen Kollokation hat also der Kollokator eine Lesart, die einen Ähnlichkeitsbezug zu seiner wörtlichen Lesart aufweist. Bei der übertragenen Verwendung wird die Bedeutung 'an eine andere Stelle gebracht werden' übernommen, jedoch ist die 'andere Stelle' nicht unbedingt wörtlich zu verstehen. Der Schüler kann gegebenfalls auch im selben Klassenraum bleiben.

Kollokationen, deren Kollokatoren metaphorisch verwendet werden, bezeichne ich im Folgenden als "metaphorische Kollokationen". Der Kollokator in metaphorischen Kollokationen ist also polysem, hat zwar in der Kollokation eine metaphorische Bedeutung, wird jedoch in einer freien Wortverbindung auch wörtlich verwendet, wie das durch das Verb versetzen in Beispiel (5) veranschaulicht wird. Diese Deutung der metaphorischen Kollokation setzt voraus, dass der Kollokator polysem ist, also mindestens zwei Lesarten hat, wodurch er je nach Kontext entweder in konkreter oder in übertragener Bedeutung verwendet wird. Metaphorische Kollokationen bilden somit eine Teilmenge der Kollokationen, in welcher die Kollokatoren in freien Wortverbindungen in wörtlicher Bedeutung, in Kollokationen jedoch in übertragener Bedeutung verwendet werden. Diese Voraussetzung schränkt zwar die Gruppe der metaphorischen Kollokatoren ein, lässt sich jedoch nicht in jedem Einzelfall problemlos anwenden. Man fragt sich z. B. bei den Wortverbindungen Sterne funkeln oder Augen funkeln, in welchem Falle das Verb wörtlich verwendet wird.

Darüber hinaus gibt es auch Kollokationen, in denen der Kollokator nichtmetaphorisch verwendet wird, wie z. B.

eine Flagge hissen, 
und solche, deren Kollokator in wörtlicher Bedeutung nicht verwendet wird. Einen nichtwörtlichen Kollokator, der in freien Wortverbindungen nicht vorkommt, veranschaulicht die folgende Kollokation:

(8) Schule schwänzen.

Die Möglichkeit der Untergliederung der Kollokationen in die Subgruppen metaphorische vs. nichtmetaphorische Kollokationen wurde in diesem Kapitel zum einen durch eine Definition und zum anderen durch Beispiele untermauert. Die Voraussetzung für die Bezeichnung metaphorische Kollokation ist dabei, dass eine Ähnlichkeitsbeziehung zwischen der konkreten und der übertragenen Lesart des Kollokators festzustellen ist. Es ist jedoch einzuräumen, dass diese Eigenschaft im Einzelfall nicht immer problemlos nachzuweisen ist. Die Überlegungen zur Metaphorik bewegen sich dabei innerhalb einer Sprache. Die Folge ist klarerweise, dass die Perspektive des Fremdsprachenlerners unberücksichtigt bleibt. Bekanntlich ist die Metaphorik für die Sprache charakteristisch, jedoch kann ihre Realisierung in verschiedenen Sprachen unterschiedlich ausfallen. Demzufolge sind die Vertereter der obigen beiden Kategorien nicht notwendigerweise deckungsgleich. Welche Ähnlichkeiten und Unterschiede sich in deutsch-ungarischer Relation feststellen lassen, wird im Folgenden beschrieben und in einem Modell erfasst.

\section{$4 \quad$ Kollokationen kontrastiv}

Im vorangehenden Kapitel ist dargestellt worden, dass eine metaphorische Kollokation vorliegt, wenn der Kollokator polysem ist und in freien Wortverbindungen in konkreter Bedeutung, in Kollokationen jedoch in übertragener Bedeutung verwendet wird. Unter dem Aspekt der Metaphorik sollen in diesem Kapitel deutsche Kollokationen und ihre Äquivalente im Ungarischen miteinander verglichen werden. Das kontrastive Modell ist in der Abbildung dargestellt. 
Abbildung

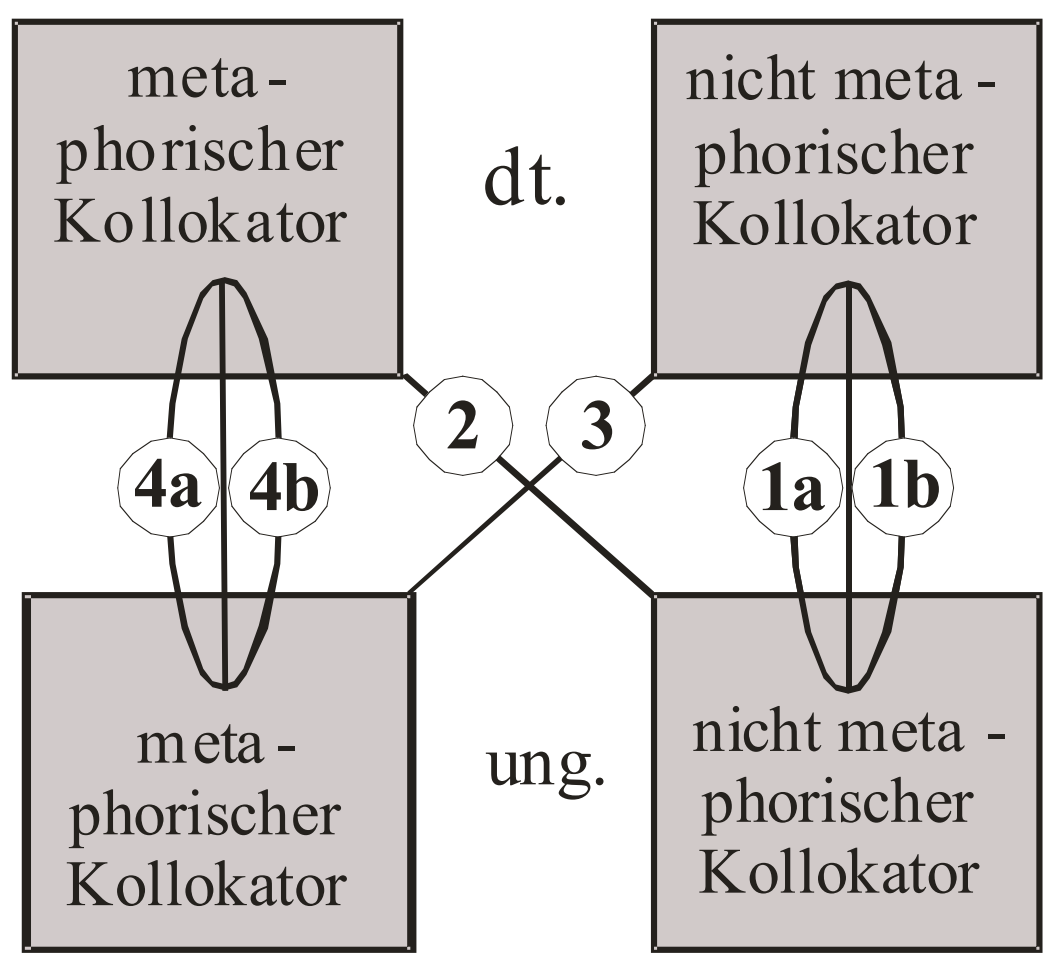

Kollokationsvergleich unter dem Aspekt der Metaphorik von Kollokatoren

Als Grundlage des Vergleichs dienen die folgenden drei Faktoren mit jeweils zwei Variablen: Es handelt sich beim Vergleich erstens um zwei Sprachen, nämlich um das Ungarische und das Deutsche. ${ }^{4}$ Zweitens kann unterschieden werden, ob eine Metaphorik vorhanden ist oder nicht. Drittens ist beim Vergleich äquivalenter Kollokationen relevant, ob die Kollokatoren in den beiden Sprachen auch gegenseitige kollokationsexterne Entsprechungen darstellen oder lediglich kollokationsinterne. Diese drei Voraussetzungen mit ihren jeweils zwei Optionen ergeben mathematisch acht mögliche Kategorien.5 Bei der Erfassung der acht Subgruppen stellt es sich jedoch heraus, dass zwei entfallen. Wenn nämlich einer metaphorischen Kollokation im Deutschen eine nichtmetaphorische Entsprechung im Ungarischen gegenübersteht, entfällt die Frage nach der kollokationsexternen Entsprechung der Kollokatoren. Ebenso in ungarisch-deutscher Relation. Die einzelnen kontrastiven Typen sind im Modell mit 1a, 1b, 2, 3, 4a, $4 \mathrm{~b}$ bezeichnet (siehe Abbildung).

Zum ersten Typ gehören jene Kollokationen, bei denen die Kollokatoren in beiden Sprachen eine nichtmetaphorische Verwendung erfahren. Innerhalb dieser Subgruppe können die Kollokatoren auch kollokationsexterne Äquivalente sein, oder lediglich kollokationsinterne.

\footnotetext{
${ }^{4}$ Die morphosyntaktischen Unterschiede der Kollokationsbestandteile werden im Modell nicht erfasst, denn sie rühren oft nicht aus ihrer Funktion - Kollokator oder Basis in einer Kollokation zu sein -, sondern es handelt sich um ihre sprachstrukturellen Eigenschaften, die sie kollokationsintern wie auch kollokationsextern aufweisen. So stellen sich in einer kontrastiven Analyse der Kollokationen hinsichtlich morphosyntaktischer Eigenschaften der Bestandteile Fragen, die sich auch im Rahmen der kontrastiven Morphologie ergeben. In deutschungarischer Relation verweise ich bezüglich kontrastiver Abhandlungen reflexiver Verben und der Artikelwörter auf Canisius (1998) und Szúcs (1999). Zu den Valenzunterschieden siehe Bassola (1995).

${ }^{5}$ Zwei hoch drei ist gleich acht.
} 


\subsection{Nichtmetaphorischer Kollokator (dt.) vs. nichtmetaphorischer Kollokator (ung.)}

\subsection{1 Äquivalente Kollokatoren}

Bei diesem Kontrasttyp handelt es sich eigentlich um totale Äquivalenz, denn sowohl die Basiswörter als auch die Kollokatoren entsprechen einander im Deutschen und Ungarischen, z. B.:

(10) die Polizei ermittelt $\approx$ a rendörség nyomoz die Polizei $=$ a rendörség; ermittelt $\rightarrow$ nyomoz

(11) Schaden verursachen $\approx$ kárt okoz $\quad$ Schaden $=$ kárt; verursachen $\rightarrow$ okoz

(12) Blumen pflücken $\approx$ virágot szed

Blumen = virágot, pflücken $\rightarrow$ szed

Die obigen Kollokationsgleichungen sind also Beispiele für vollständige Äquivalenz, jedoch ist anzumerken, dass die Äquivalenz der Kollokationsbestandteile nicht beidseitig ist. Das lässt sich am ehesten in Beispiel (12) erkennen. Das Verb pflücken ist zwar äquivalent mit szed, jedoch ist das ungarische Verb nicht auf einige wenige Substantive beschränkt wie das deutsche. So hätte ung. szed im Deutschen mehrere Entsprechungen. ${ }^{6}$

\subsubsection{Nichtäquivalente Kollokatoren}

Bei dem Kontrasttyp 1b) geht es weiterhin um nichtmetaphorische Kollokatoren, jedoch sind diese im Deutschen und Ungarischen kollokationsextern nicht äquivalent, sie stehen also in partieller Äquivalenz zueinander. Beispiele für diesen Kontrasttyp sind etwa:

(13) bespielte Kassette

(14) sich die Zähne putzen

(15) das Schiff kentert

$$
\begin{aligned}
& \text { Koll. (ung.) } \\
& \approx \text { müsoros kazetta } \\
& \approx \text { fogat mos } \\
& \approx \text { a hajó felborul }
\end{aligned}
$$

Nachdem die vertikale Relation der zweiten Spalte im Modell durch einige Besipiele veranschaulicht worden ist, folgen die diagonalen Relationen 2) und 3), bei denen jeweils in der einen Sprache der Kollokator metaphorisch verwendet wird, in der anderen jedoch in konkreter Bedeutung.

\subsection{Metaphorischer Kollokator (dt.) vs. nichtmetaphorischer Kollokator (ung.)}

(15) blutiger Anfänger

(16) Schmerz stillen
Koll. (ung.)

$\approx$ teljesen kezdö

₹fájdalmat enyhít
Spiegelübersetzung

"totaler Anfänger"

"Schmerz erleichtern"

Die wörtliche Übersetzung der Kollokation blutiger Anfänger im Beispiel (15) durch totaler Anfänger ergibt in diesem Fall eine Wortverbindung, die auch im Deutschen gebräuchlich ist. Das Beispiel soll jedoch veranschaulichen, dass eine metaphorische Kollokation im Deutschen ein nichtmetaphorisches Äquivalent im Ungarischen hat. Die gemeinsame Entspre-

\footnotetext{
${ }^{6}$ In den Beispielen (10)-(12) gibt der Pfeil $(\rightarrow$ ) die Äquivalenzrichtung an.

${ }^{7}$ Wort- für-Wort Übersetzung der ungarischen Kollokation.
} 
chung von teljesen kezdö für totaler Anfäger und blutiger Anfänger 8 ist gleichzeitig ein Beleg für die Unterdifferenzierung im Ungarischen.

\subsection{Nichtmetaphorischer Kollokator (dt.) vs. metaphorischer Kollokator (ung.)}

Unter dem Aspekt der Metaphorik lässt sich im Modell ein weiterer Kontrasttyp in diagonaler Relation erfassen, nämlich Typ 3. Diese Untergruppe stellt eigentlich eine inverse Kategorie zum Typ 2) dar, bei dem nichtmetaphorisch gebrauchte deutsche Kollokatoren Entsprechungen in übertragener Bedeutung im Ungarischen haben. Beispiele für den Kontrasttyp 3) nichtmetaphorischer (dt.) vs. metaphorischer Kollokator (ung.) sind die Kollokationsgleichungen (17)-(19). ${ }^{9}$

(17) unbeschwerte Kindheit

(18) Geld verdienen

(19) in Panik geraten
Koll. (ung.)

$\approx$ felhötlen gyermekkor

$\approx$ pénzt keres

₹pánikba esik
Spiegelübersetzung

"wolkenlose Kindheit"

"Geld suchen"

"in Panik fallen"

Außer den diagonalen Relationen gibt es im Modell symmetrisch zu den Typen 1 a) und 1b) noch weitere vertikale Relationen. Jene Kollokationsgleichungen sind im Modell in der ersten Spalte erfasst, in denen die Kollokatoren in beiden Sprachen metaphorisch verwendet werden. Je nachdem, ob die Metaphorik im Deutschen und Ungarischen äquivalent oder unterschiedlich realisiert ist, gibt es zwei Untergruppen, äquivalente und nichtäquivalente Kollokatoren.

\subsection{Metaphorischer Kollokator (dt.) vs. metaphorischer Kollokator (ung.)}

\subsection{1 Äquivalente metaphorische Kollokatoren}

(20) Applaus ernten $\approx$ tapsot arat

(21) tiefe Trauer $\approx$ mély gyász

(22) eine Epidemie bricht aus ₹járvány tör ki

(23) ein Problem taucht auf $\approx$ felmerül egy probléma

(24) grober Fehler $\approx$ durva hiba

(25) Durst löschen $\approx$ szomjat olt

(26) die Meinung teilen $\approx$ osztja a véleményt

(27) eine Freundschaft pflegen $\approx$ barátságot ápol

(28) gepfefferte Preise $\approx$ borsos árak

(29) im Internet surfen $\approx$ szörföl az interneten

\footnotetext{
${ }^{8}$ Scherfer nennt Kollokationen wie in Beispiel (15) lexikalische Kollokationen (vgl. Scherfer 2001: 12).

${ }^{9}$ Die Metaphorik im Ungarischen ist in der dritten Spalte der Kollokationsgleichung durch die wörtliche Übersetzung ins Deutsche angedeutet.
} 
Die Beispiele (20)-(29) sind weitere Belege für die totale Äquivalenz zwischen deutschen und ungarischen Kollokationen, jedoch handelt es sich hier im Gegensatz zu den Kollokationsgleichungen (10)-(12) um metaphorisch verwendete Kollokatoren. ${ }^{10}$

Die gemeinsame Metaphorik der Ausgangs- und der Zielsprache ist für einen Deutschlerner eigentlich lernfördernd, denn der Bedeutungstransfer aus dem Ungarischen führt in diesem Falle zu üblichen Wortverbindungen, im Gegensatz zum Ausgangsbeispiel eine Lehrveranstaltung <aufnehmen>. Dabei stellt sich die Frage für den Lerner, woher denn die Gemeinsamkeit der Übertragungen rührt, und vielleicht auch der Wunsch, möglichst viel totale Äquivalenz in der Zielsprache anzutreffen, denn der Rückgriff auf die Verbindbarkeitsregeln der Wörter in der Erstsprache erweist sich bei diesen Kollokationsgleichungen als erfolgreiche Strategie. Die gemeinsame Metaphorik in zwei Sprachen kann bekanntlich auch außersprachlich bedingt sein, indem sie einen Kulturkontakt widerspiegelt, wie in Beispiel (28). Auch Entlehnungen von heute wie das Beispiel (29) im Internet surfen sind "lernerfreundliche" Kollokationen.

Auf die Frage, inwieweit universelle Metaphorisierungsprozesse in den Sprachen nachzuweisen sind, bietet die kognitive Linguistik ansatzweise Antworten. Zur Zeit ist jedoch der Lerner mit der Tatsache im Erwerbsprozess konfrontiert, dass die Metaphorik der Kollokatoren im Deutschen von jener im Ungarischen abweichen kann, wie auch in den Beispielen (30)-(35): ${ }^{11}$

\subsubsection{Nichtäquivalente metaphorische Kollokatoren}

$\begin{array}{lll}\quad \text { Koll. (dt.) } & \text { Koll. (ung.) } & \text { Spiegelübersetzung } \\ \text { (30) dickes Lob } & \approx \text { komoly dícséret } & \text { "ernstes Lob" } \\ \text { (31) jemandem ins Wort fallen } & \approx \text { szavába vág } & \text { "jemandem ins Wort schneiden" } \\ \text { (32) dicke Freunde } & \approx \text { közeli barátok } & \text { "nahe Freunde" } \\ \text { (33) die Gemüter erhitzen } & \approx \text { a kedélyeket felborzol } & \text { "die Gemüter sträuben" } \\ \text { (34) auf die Idee kommen } & \approx \text { ötlete támad } & \text { "die Idee attakiert jemanden" } \\ \text { (35) in Wettbewerb treten } & \approx \text { versenybe száll } & \text { "in Wettbewerb fliegen" }\end{array}$

Die Beispiele (30)-(35) sind Belege dafür, dass in Kollokationen der Kollokator metaphorisch verwendet wird. Jedoch fallen die Übertragungen im Deutschen und im Ungarischen unterschiedlich aus. Wie gehen Fremdsprachenlerner mit diesen Unterschieden um? Welche Kompensationsstrategien setzen sie ein, wenn es sich um metaphorische und welche, wenn es sich um nichtmetaphorische Kollokationen handelt?

\footnotetext{
${ }^{10}$ Die Kollokatoren sind auch in konkreter Bedeutung äquivalent zu einander, wie etwa in Getreide ernten $\approx$ gabonát arat; tiefer Graben $\approx$ mély árok; ein Tier bricht aus $\approx$ kitör egy állat; das $U$-Boot taucht auf $\approx$ felszinre jön a tengeralattjáró; grober Stoff $\approx$ durva szövet; Feuer löschen $\approx$ tüzet olt; den Kuchen teilen; den Kranken pflegen $\approx$ beteget ápol.

11 Der metaphorisch gebrauchte Kollokator ist wörtlich übersetzt, um die Metaphorik des ungarischen Kollokators im Deutschen zu zeigen.
} 


\section{Empirische Untersuchung zum Transferverhalten von Deutschlernern ungari- scher Muttersprache}

Im Kapitel 4 ist beschrieben worden, dass die Äquivalenz der Kollokationen in zwei Sprachen nicht automatisch mit der Äquivalenz der Kollokatoren einhergeht. Inwieweit beeinträchtigt dieses Faktum die Akzeptabilität der Schüleräußerungen? Das Fehlerbeispiel eine Lehrveranstaltung <aufnehmen> in Kapitel 1 war z. B. keine korrekte Formulierung, doch hat sie letztendlich die Kommunikation nicht verhindert. Zur geglückten Verständigung haben vermutlich neben dem Kontext auch die Ungarischkenntnisse der Lehrkraft beigetragen. Der Rezipient hat wohl die wörtliche Übersetzung erkannt und dadurch die Sprechabsicht des Studenten richtig deuten können.

\section{$5.1 \quad$ Hypothese}

In diesem Zusammenhang stellt sich die Frage zum einen, wie häufig Lerner bei der Bildung von Kollokationen auf ihre L1 zurückgreifen. Zum anderen ist zu fragen, ob der Rückgriff auf die L1 bei den einzelnen kontrastiven Kollokationstypen unterschiedlich häufig sei. Diese Fragestellungen bilden die Grundlage zur folgenden Hypothese:

Nichtmetaphorische Kollokatoren werden mehr aus der L1 transferiert als metaphorische Kollokatoren. Eine andere Formulierung der Hypothese wäre, dass die Lerner bei metaphorisch gebrauchten Kollokatoren weniger aus der L1 transferieren als bei nichtmetaphorischen.

\subsection{Empirische Datenerhebung}

Zur Überprüfung der Hypothese sind Deutschlerner aus unterschiedlichen Schultypen herangezogen worden, und zwar DaF-Abiturienten, Deutsch-als-Minderheitensprache(DaM)Abiturienten sowie Germanistikstudenten (GeS). Alle Probanden (200 Versuchspersonen pro Lernerstufe) haben Ungarisch als Muttersprache und Deutsch als erste Fremdsprache angegeben. Der Sprachstand der Lerner im Deutschen setzt auf der Stufe A2 ${ }^{12}$ ein und stellt ein gewisses Stufen-Kontinuum dar, bis er schließlich mit C1 endet. Abhängig von der Zahl ihrer Lernjahre und ihrer Wochenstundenzahl sowie ihrer jeweiligen Sprachbiographie decken DaF-Gymnasiasten ${ }^{13}$ in etwa die Stufen von A2 bis B2, vereinzelt auch C1 ab. Die Anforderungen der Rahmenrichtlinien für das DaF-Abiturnin Gymnasien sind in etwa vergleichbar mit dem Kenntnisstand B2. ${ }^{14}$

Die zweite Zielgruppe sind DaM-Abiturienten. Diese Schüler ${ }^{15}$ haben im Gymnasium zweisprachigen, deutsch-ungarischen Unterricht. Zweisprachiger Unterricht bedeutet, dass mindestens in der Hälfte der Fächer die Unterrichtssprache Deutsch ist. Der deutschsprachige

\footnotetext{
12 Nach der Einstufung des "Gemeinsamen Europäischen Referenzrahmens" werden bekanntlich für Fremdsprachenkenntnisse die folgenden aufeinader aufbauenden Niveaus vorgeschlagen: A1, A2, B1, B2, C1 und C2.

${ }^{13}$ Die Versuchspersonen sind DaF-Abiturienten im Schuljahr 2001/2002, vorwiegend aus Südtransdanubien.

${ }^{14}$ Den Vergleich konnte ich nicht direkt durchführen, weil die Rahmenrichtlinien die Themen zwar ausführlich aufreihen, aber kein Glossar beilegen.

15 Die Versuchspersonen sind DaM-Abiturienten im Schuljahr 2001/2002 in Baja, Budapest, Pécs, Gyönk. Zu ihrer Sprachattitüde vgl. Gerner 2001.
} 
Fachunterricht wird noch durch Sprachunterricht ergänzt, in dessen Rahmen in fünf Wochenstunden deutsche Literatur und Grammatik unterrichtet werden. Der Unterricht wird zum Teil von Muttersprachlern durchgeführt. Die schulische Sprachvermittlung wird durch Schüleraustausch mit Österreich und Deutschland vertieft. In den Unterricht werden Nationalitäteninhalte integriert, so lernen die Schüler ungarndeutsche Literatur und Volkskunde kennen. Sie gewinnen einen Einblick in die Geschichte und Bräuche der ungarndeutschen Volksgruppe und können in außerschulischen Tätigkeiten die Möglichkeit wahrnehmen, die Volkskultur zu pflegen (vgl. Wild 1992). Die Sprachkenntnisse der Abiturienten werden je nach Note im Abitur nach den ungarischen Einstufungen, Mittelstufe bzw. Oberstufe bescheinigt. Ein Großteil der Schüler erwirbt auch das "Deutsche Sprachdiplom Zweiter Stufe". ${ }^{16}$ So reicht ihr Niveau etwa von B2 bis C1.

Die dritte Zielgruppe sind GeS ${ }^{17}$ aus höheren Jahrgängen. Ihre Deutschkenntisse, die auf der Oberstufe anzusiedeln sind, haben sie mit dem erfolgreichen Bestehen des ersten sprachlichen Rigorosums an der Universität unter Beweis gestellt. Ihre Einstufung nach den Vorgaben des Gemeinsamen Europäischen Referenzrahmens wäre in etwa zwischen B2 bis C1 anzusiedeln.

Die Datenerhebung erfolgte durch Übersetzungsaufgaben, in denen die Basis angegeben war und der Kollokator abgefragt wurde, in der Form wie das das folgende Item veranschaulicht: eine Ehe ........ ₹ házasságot kött ${ }^{18}$

Die Kollokationen in den 40 Items stammen aus Glossaren in Materialien, die auf das Abitur bzw. auf Sprachprüfungen vorbereiten. In den Test sind Kontrasttypen wie in Kapitel 4 beschrieben, aufgenommen worden.

\subsection{Datenanalyse}

Bei der Auswertung der Testitems werden positive und fehlende Antworten sowie Normverstöße statistisch erfasst und negative Antworten mit Hilfe der Fehleranalyse interpretiert. Dabei werden die Fehler erst einmal identifiziert und unter ihnen diejenigen bestimmt, die sich auf negativen-L1-Transfer zurückführen lassen. ${ }^{19}$

\subsubsection{Durch negativen L1-Transfer bedingte Fehler}

Bei der Auswertung des Items stellt sich die Frage, welche Fehler mit einem Rückgriff auf die L1, auf das Ungarische erklärt werden können. Wortverbindungen werden im Belegmaterial dann als Ergebnisse eines negativen L1-Transfers betrachtet, wenn anzunehmen ist, dass sie durch eine direkte, nachvollziehbare Übernahme aus dem Ungarischen entstanden sind. Dabei können für den negativen L1-Transfer in Anlehnung an Hufeisen/Neuner (2000: 26) zwei

\footnotetext{
16 Das Deutsche Sprachdiplom Zweiter Stufe wird von der Ständigen Konferenz der Kultusminister der Länder in der Bundesrepublik Deutschland vergeben und gilt als Nachweis der Deutschkenntnisse, die für die Aufnahme eines Hochschulstudiums in Deutschland erforderlich sind.

17 Die Versuchspersonen sind GeS im Schuljahr 2001/2002 in Budapest, Pécs, Szeged und Veszprém.

18 Lösung: eine Ehe schliessen $\approx$ házasságot köt.

${ }^{19} \mathrm{Zu}$ einer Typologie der Fehlerebenen vgl. Katsikas (2002).
} 
Typen, nämlich Substitution ${ }^{20}$ und Unterdifferenzierung unterschieden werden. Für Substitution eines Kollokators aus dem Ungarischen als Fehlerursache konnten im Fehlerkorpus keine Belege gefunden werden. Auf Unterdifferenzierung ${ }^{21}$ als Fehlerursache lassen sich mehrere Items zurückführen. Da das Verb vezet mehrere Enstprechungen im Deutschen hat,

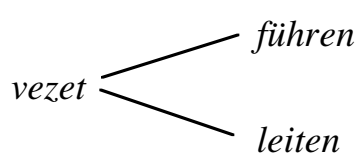

bildet diese Interferenz eine weitere L1-bedingte Fehlerquelle, wie z. B.

einen Haushalt <leiten> $\approx$ háztartást vezet ( $\approx$ einen Haushalt führen).

Der Rückgriff auf die L1 ist im Falle der Unterdifferenzierung eine leicht irreführende Strategie, denn die L1 kann bei der Wahl keine Hilfestellung geben, wirkt bei der Wortwahl eher störend. Eine weitere Unterdifferenzierung vom ungarischen Verb vigyáz, in der Bedeutung von 'achten/aufpassen', ist in den folgenden Lösungen für auf die Figur achten zu finden, nämlich auf die Figur <aufpassen>. Weitere Belege sind im Test die Verwechslung von bringen und holen sowie von den Adjektiven düster, dunkel, finster. Der Fehler im Item törvényt hoz in der Lösung ein Gesetz <holen> ist durch die Unterdifferenzierung des ungarischen Verbs hoz gegenüber holen/bringen zurückführen. Dieser Fehlertyp ist auch im Item ein Kind auf die Welt bringen häufig in Form der Lösung ein Kind in die Welt <holen> zu beobachten.

Es gibt darüber hinaus noch Fehler im Fehlerkorpus, deren Zustandekommen das Ungarische nachweisbar beeinflusst, die jedoch nicht von den obigen zwei Kategorien erfasst werden können. Ein Eintrag eines Probanden im Item wird als weiterer negativer L1-Transfer gedeutet, wenn eine wortwörtliche Übersetzung des Kollokators aus dem Ungarischen ins Deutsche nachvollziehbar ist, obwohl der Rückgriff auf die L1 eine im Deutschen nicht usuelle Wortverbindung hervorbringt, wie z. B:

die Uhr $<$ eilt $>/<$ beeilt sich $>\approx$ siet az óra statt die Uhr geht vor.

Solche Fehler entstehen also durch die Übersetzung des Kollokators aus dem Ungarischen, so kann dabei die Fehlerursache Spiegelübersetzung genannt werden. Beispiele für Spiegelübersetzungen sind der Tabelle 1 zu entnehmen.

\footnotetext{
20 Das Phänomen wird auch mit dem Terminus Direktübernahme bezeichnet wie z. B. in Näf (2001).

${ }^{21}$ Der Terminus Unterdifferenzierung ist bekanntlich polysem. Er bedeutet zum einen ein sprachliches Phänomen und zum anderen eine mentale Operation des Sprechers. Unterdifferenzierung als sprachliches Phänomen bezieht sich auf kontrastiv ermittelte Gegebenheiten der L1 und L2, nämlich dass eine Wortform z. B. des Ungarischen zwei oder mehr Unterbedeutungen umfasst, die im Deutschen auf verschiedene Wortformen verteilt sind. Unterdifferenzierung als Transfer meint, dass der Lerner letztendlich die Wörter verwechselt, die im Vergleich zur L2 in der L1 unterdifferenziert sind. Im obigen Text handelt es sich um die mentale Operation.
} 


\begin{tabular}{|l|l|l|l|}
\hline & $\begin{array}{l}\text { DaF- } \\
\text { Abiturienten }\end{array}$ & $\begin{array}{l}\text { DaM- } \\
\text { Abiturienten }\end{array}$ & $\begin{array}{l}\text { Germanistik- } \\
\text { Studenten }\end{array}$ \\
\hline $\begin{array}{l}\text { jemandem ins Wort }<\text { schneiden }>: \\
\text { (= jemandem ins Wort fallen) }\end{array}$ & $76^{22}$ & 40 & 33 \\
\hline $\begin{array}{l}\text { in Wettbewerb }<\text { steigen/einsteigen/fliegen }>: \\
(=\text { in Wettbewerb treten })\end{array}$ & 70 & 48 & 40 \\
\hline $\begin{array}{l}\text { eine Ehe }<\text { binden/ verbinden }>: \\
(=\text { eine Ehe schließen) }\end{array}$ & 75 & 20 & 10 \\
\hline $\begin{array}{l}\text { Bücher }<\text { fressen/essen }>: \\
(=\text { Bücher verschlingen })\end{array}$ & 40 & 28 & 22 \\
\hline $\begin{array}{l}\text { das Bett }<\text { drücken/pressen>: } \\
(=\text { das Bett hüten })\end{array}$ & 74 & 53 & 40 \\
\hline $\begin{array}{l}\text { in Panik <fallen>: } \\
(=\text { in Panik geraten })\end{array}$ & 122 & 51 & 43 \\
\hline
\end{tabular}

Tabelle 1: Spiegelübersetzungen des metaphorischen Kollokators aus der L1 in Lernerproduktionen

\subsubsection{Hypothesenüberprüfung}

Die empirischen Daten untermauern die Hypothese für die DaM-Abiturienten und für die GeS. Das bedeutet, sie haben Kollokatoren in metaphorischer Lesart deutlich seltener wörtlich aus der L1 in die Fremdsprache übersetzt als nichtmetaphorische Kollokatoren. Für DaFAbiturienten konnte kein Unterschied festgestellt werden. Sie stützen sich sowohl bei den metaphorischen wie bei den nichtmetaphorischen Kollokationen weitgehend auf ihre L1 (vgl. Tabelle 2). Die empirischen Daten belegen, dass DaM-Abiturienten und GeS bei den gestellten Testaufgaben die Metaphorik der Wörter als markiert ${ }^{23}$ empfinden. Sie gehen nämlich häufig davon aus, dass die metaphorischen Kollokationen aus dem Ungarischen nicht transferierbar sind. Da die ermittelten Werte die Hypothese für die Testgruppe der DaF-Abiturienten nicht bestätigen, kann angenommen werden, dass die Metaphorik der Kollokatoren für diese Zielgruppe, also auf dem Niveau etwa B2, die Strategie des Rückgriffs auf die Muttersprache nicht beeinträchtigt.

\footnotetext{
${ }^{22}$ Die Zahlen zeigen die Vorkommenshäufigkeit der Wortverbindung im Test unter 200 Probanden je Zielgruppe.

${ }^{23}$ Gemeint ist hier der Markiertheitsbegriff im Sprachvergleich. Der Begriff bezeichnet das Phänomen, dass Lerner durch den Vergleich der L1 und L2 die L1-Transferierbarkeit einschätzen (vgl. Edmondson/House 2000: 145-146).
} 


\begin{tabular}{|l|l|l|l|}
\hline & DaF-Abiturienten & DaM-Abiturienten & Germanistik-Studenten \\
\hline $\begin{array}{l}\text { Anteil der Fehler durch } \\
\text { negativen L1-Transfer in } \\
\text { metaphorischen Kolloka- } \\
\text { tionen }\end{array}$ & $38 \%$ & $20 \%$ & $16 \%$ \\
\hline $\begin{array}{l}\text { Anteil der Fehler durch } \\
\text { negativen L1-Transfer in } \\
\text { nichtmetaphorischen } \\
\text { Kollokationen }\end{array}$ & $39 \%$ & $33 \%$ & $30 \%$ \\
\hline
\end{tabular}

Tabelle 2: Daten zur Überprüfung der Metaphorik-Hypothese

Sehen wir uns dazu noch Einträge zu vollständig äquivalenten Kollokationen mit metaphorischem Kollokator an, wie z. B. das Item gepfefferte Preise. Das Belegmaterial lässt sich als das Ergebnis einer negativen Einstellung der Studenten gegenüber der Transferierbarkeit des metaphorischen Kollokators interpretieren. Eine große Zahl der Lerner hat die Aufgabenstellung des Items durch synonyme Adjektive, wie hoch, groß, teuer gelöst (DaF: 117, DaM: 108, GeS: 85). Eine ähnliche Einstellung der Lerner ist im Item einen stürmischen Applaus ernten zu beobachten, da die positiven Einträge vorwiegend mit dem Verb bekommen gelöst wurden. Das Einsetzen dieser Vermeidungsstrategien, der Rückgriff auf die L2, lässt die Schlussfolgerung zu, dass Lerner den metaphorischen Kollokator als markiert empfinden und dazu tendieren, die Aufgabe zu vereinfachen. Wenn davon ausgegangen wird, dass das Substantiv Pfeffer aus dem Grundwortschatz bekannt sein müsste, sind auch die Nulleinträge mit hoher Wahrscheinlichkeit eher als Nichttransferierbarkeitsannahme und weniger als Abrufbarkeitsstörung zu deuten.

Im Item scharfer Gegensatz, handelt sich um eine Kollokation mit einem Kollokator, scharf, ${ }^{24}$ der in denotativem Gebrauch zum Grundwortschatz gehört, und deshalb den Probanden bekannt sein müsste. Auch hier spricht die den durchschnittlichen L2-Transfer übersteigende Zahl für die Annhame der Nichttransferierbarkeit von scharf in metaphorischer Bedeutung. Wir wissen natürlich weiterhin nicht, wie oft ein L1-Transfervorhaben wegen einer Kenntislücke oder Abrufbarkeitsstörung nicht erfolgt ist. Bei Verben wie lóg ( $\approx$ hängen), die zum Grundwortschatz gehören, können wir davon ausgehen, dass sie den meisten Probanden bekannt sind. Es gibt trotzdem nur einige wenige Belege für die wortwörtliche Übersetzung des Kollokators lóg in iskolából lóg ( $\approx$ die Schule schwänzen):

die Schule <hängen>: DaF: 24, DaM: 2, GeS: - .

\footnotetext{
${ }^{24}$ Scharf ist sogar durch das englische sharp gestützt, so haben wir ein zweites Argument dafür, mit großer Sicherheit sagen zu können, dass dieses Adjektiv den meisten Probanden bekannt sein muss.
} 


\section{Didaktische Konsequenzen}

Aus der Analyse des empirischen Materials geht hervor, dass DAF-Abiturienten die Metaphorik der Kollokatoren eher als nichtmarkiert empfinden und sich mit Vorliebe der Strategie des L1-Transfers bedienen. Die ermittelten Werte belegen, dass sich erst im Laufe des Erwerbsprozesses allmählich eine Tendenz zur Annahme der Nichttransferierbarkeit entwickelt. Es gibt jedoch im Fehlerkorpus auch dafür Beispiele, dass Fortgeschrittene auf dem Niveau C1 die Kompatibilitätsregeln ihrer L1 auf die Fremdsprache übertragen. Wie dem kontrastiven Modell zu entnehmen ist, wird der Lerner im Input mit beiden Möglichkeiten konfrontiert. Kollokatoren können häufig in der Ausgangs- und in der Zielsprache äquivalent sein. Es gibt jedoch auch reichlich Beispiele auch für das Gegenteil. Das führt dazu, dass der Lerner nur schwierig Regularitäten ableiten kann. Er muss damit rechnen, dass die Transferierbarkeit der Kollokatoren unvorhersagbar ist.

Welche Hilfe kann der Unterricht für den Lerner beim Aneignen der Kollokationen trozdem leisten? Es dürfte lernfördernd wirken, wenn die Existenz von Kollokationen als wortübergreifende Einheiten des Wortschatzes, an der Schnittstelle zwischen Idiomen und freien Wortverbindungen, bewusst gemacht wird. Eine spezifische Aufgaben- und Übungstypologie für die explizite Schulung der Kollokationen im DaF-Unterricht in drei Phasen analog zur Wortschatzdidaktik, könnte wie etwa folgt aussehen:

1) Entdecken der Kollokationen als Einheiten

2) Einüben der Kollokationen als Einheiten

3) Anwenden der Kollokationen als Einheiten. ${ }^{25}$

Es ist zu hoffen, dass die genannten Schritte den Erwerbsprozess optimieren und gleichzeitig die Strategiepalette des autonomen Lernens erweitern können. Es ist bezüglich der metaphorischen Kollokation zu überlegen, welche metapherspezifischen Übungen in der zweiten Phase der nachhaltigen Einprägung der Kollokationen dienen könnten. Im Folgenden werden zu dieser Fragestellung Vorschläge unterbreitet und ihre Anwendungsmöglichkeiten exemplarisch dargestellt.

\section{1 Überlegungen zur Motivation der Metaphorik}

\subsubsection{Bewusstmachung von Kulturkontakt}

Da die Äquivalenz der Kollokatoren im Deutschen und Ungarischen unvorhersagbar ist, müssen sowohl total als auch partiell äquivalente Kollokationen in Übungen berücksichtigt werden. Die Bewusstmachung der gegenseitigen Entsprechung der Kollokatoren kann z. B. dadurch unterstützt werden, dass Kulturkontakt und Entlehnung bei der jeweiligen Kollokation explizit angesprochen werden. Das Nachvollziehen der Bedeutungserweiterung des Kollokators, eingebettet in die Kulturgeschichte wird wohl bei Lernern auf Interesse stoßen und dadurch die Gedächtnisleistung fördern. Sinntragende Kontexte, die gleichzeitig mit der neuen Wortform auch neue Inhalte vermitteln, bewegen das Vokabellernen in Richtung mitteilungsbezogene Kommunikation. So könnte z. B. die vollständig äquivalente Kollokati-

\footnotetext{
${ }^{25}$ Zur ausführlichen Beschreibung der drei Phasen der Kollokationsvermittlung siehe Reder (2002).
} 
onsgleichung gepfefferte Preise ₹ borsos árak mit Hilfe ihrer Etymologie dargestellt und eingeübt werden. Die Kollokation gepfefferte Preise verweist bekanntlich auf den Wert der Gewürze in Europa. Die wichtigste und älteste Gewürzpflanze Indiens, der Pfeffer, der auch schon im antiken Griechenland und Rom hochgeschätzt und im Mittelalter mit Gold gleichgestellt war, hat seinen hohen Wert in der obigen Kollokation manifestiert. Er diente häufig als Zahlungsmittel. Für den Stellenwert des Pfeffers spricht z. B., dass der gotische König Alarich (408 n.Chr.) vom belagerten Rom als Lösegeld Gold, Silber und Pfeffer forderte. Die Synonymie zwischen hohe Preise und gepfefferte Preise lässt sich also diachron erklären.

Auch die kognitive Semantik bietet für einige Kollokationen eine nachvollziehbare Erklärung zur Motivation der jeweiligen Bedeutungsübertragung an. Durch diese Einsichten in die Motivation der Bedeutungsübertragung könnte die Aneignung der Kollokationen im Einzelfall begünstigt werden.

\subsubsection{Anwendung der Erkenntnisse der kognitiven Semantik}

Im Rahmen der kognitiven Semantik zeigten Lakoff und Johnson (1980), dass Metaphern in der Sprache allgegenwärtig sind, wenn auch in einer unauffälligen und dem Sprachträger nicht bewussten Form. Mit Hilfe der Beispiele hohle Phrasen, offene Worte, leeres Geschwätz lässt sich dem Lerner erklären, dass die Attribute in diesen Beispielen nicht wörtlich gemeint sein können. Diese Wortverbindungen lassen exemplarisch nachvollziehen, wie in der Sprache Konzepte aus dem unmittelbaren Erfahrungsbereich des Menschen auf Abstrakta übertragen worden sind. Wir wissen aus Erfahrung, da wir es sehen können, dass ein konkreter Gegenstand, in dem man etwas aufbewahren kann, hohl, offen oder leer ist. Diese physisch fassbaren Eigenschaften wurden auf Abstrakta übertragen. Die Metapher in den oben angeführten Beispielen beruht auf dem Konzept eines Behälters, denn "Wörter sind Behälter", was dann das Abstraktum Sprache strukturiert. Wenn wir die Beispiele zum Konzept "Wörter sind Behälter" (vgl. Baldauf 1997: 15) ins Ungarische übersetzen, lässt sich jeweils eine vollständige Äquivalenz bei den obigen Kollokationen feststellen. Es gibt wohl zahlreiche Äquivalenzen im Deutschen und im Ungarischen, die auf die gemeinsame konzeptuelle Metaphorik zurückzuführen sind. Die Miteinbeziehung des Konzeptes zur Erklärung der Verbindbarkeit der Wörter könnte wohl bei der Aneignung einer Reihe von Kollokationen hilfreich sein. Die Anwendung der konzeptuellen Metaphorik bei der Kollokationsvermittlung ist jedoch nicht ohne Ausnahmen. Bei den folgenden Kollokationsgleichungen z. B.:

$$
\begin{aligned}
& \text { leise Hoffnung } \approx \text { halvány remény } \quad(<\text { blasse }>\text { Hoffnung }) \\
& \text { leise Ahnung } \quad \approx \text { halvány sejtelem } \quad(<\text { blasse }>\text { Ahnung) }
\end{aligned}
$$

beruht die Metaphorik im Deutschen wie im Ungarischen auf dem Konzept der "schwachen Wahrnehmbarkeit". Diese Art der Wahrnehmbarkeit abstrakter Dinge wird im Deutschen jedoch mit auditiven und im Ungarischen mit visuellen Adjektiven realisiert. Die Tatsache, dass Konzepte in den einzelnen Sprachen nicht durchgehend gleich realisiert werden, erschwert natürlich die Einbeziehung des Konzeptes bei der Kookkurrenzerklärung für Lerner. Die Aufgabe an den Lerner, auch bei unterschiedlicher Realisierung das gemeinsame Konzept 
zu entdecken, kann m. E. durch dessen Aktivität bei einer Problemlösung nachhaltig dazu beitragen, dass er diese Kollokation in seinem Langzeitgedächtnis speichert.

\subsubsection{Die Bewusstmachung der Polysemie des Kollokators}

Zur Förderung der Behaltensleistung könnte auch die Aufgabe beitragen, dass ein metaphorischer Kollokator mit seiner wörtlichen Lesart in Bezug gesetzt werden sollte. Dabei ist ein gemeinsames Sem, eine Ähnlichkeitsbeziehung, zu entdecken, welche die Bedeutungsübertragung erklärt. Bei der Erprobung dieses Aufgabentyps haben Lerner z. B. für die Kollokation dicke Freunde den Bezug zum dicken Seil hergestellt und als gemeinsames Merkmal 'die Haltbarkeit' ermittelt.

\section{$7 \quad$ Ausblick}

Die Analyse des empirischen Datenmaterials, das durch ein Testverfahren gewonnen wurde, lässt die Schlussfolgerung zu, dass Lerner im Erwerbsprozess ihre Einstellung zur Transferierbarkeit der metaphorischen Kollokatoren ändern. Ein Rückgriff auf die L1 wird in Kollokationen häufig nicht eingesetzt, obwohl diese Strategie in manchen Fällen zum positiven Transfer führen würde. Als mögliche Weiterführungen der Überlegungen könnte sich ein nächtster Schritt ergeben, nämlich die Überprüfung der Hypothese auf der Grundlage von neuem Datenmaterial, das sich z. B. aus freien Aufsätzen oder anderen Testaufgaben erstellen lässt. Darüber hinaus bietet sich eine weitere Fragestellung an, nämlich bei Lernern, die Deutsch als zweite Fremdsprache erlernen, den Einfluss der ersten Fremdsprache auf das Transferverhalten zu untersuchen. Es ist möglich, dass das Transferverhalten, das bei den Testpersonen der vorliegenden Arbeit zu beobachten war, nicht lediglich für diese Probanden und nicht nur für Lerner ungarischer Muttersprache charakteristisch ist, sondern für Fremdsprachenlerner im Allgemeinen. ${ }^{26}$ Solche empirische Untersuchungen können dazu beitragen, dass man mehr über das Strategieverhalten der Lerner erfährt. Der erklärte Zweck ist letztendlich, den Erwerbsprozess durch geeignete Aufgaben und Übungen zu unterstützen, sowie weitere Lern- und Kompensationsstrategien zu erarbeiten. Dabei könnte eine Wortschatzvermittlung, die Kollokationen als Wortschatzeinheiten unter kontrastivem Aspekt fokussiert lernfördern wirken. Der vorliegende Aufsatz versteht sich als ein Plädoyer für die Relevanz der Schulung von Kollokationen als lexikalischer Einheit zwischen freien Wortverbindungen und Idiomen im Deutschunterricht. Das Ziel dabei ist, dass Lerner das Deutsche nicht lediglich auf einem bestimmten Niveau sprechen können, sondern, dass sie das Deutsche auch beherrschen.

\footnotetext{
26 Die Übertragung der Polysemie in der L1 auf die Zielsprache ist ein häufig zu beobachtendes Phänomen. Eine Beschreibung sowie Belege für das Sprachenpaar und die Übernahmerichtung französisch-englisch sind z. B. dem Aufsatz von Näf/Pfander (2001) zu entnehmen.
} 


\section{Literaturangaben}

Baldauf, Christa (1997): Metapher und Kognition. Grundlagen einer neuen Theorie der Alltagsmetapher. Frankfurt.

Bassola, Péter (1995): "Kontrastive Substantivvalenz in Forschung und Unterricht". In: Popp, Heidrun (ed.): Deutsch als Fremdsprache. An den Quellen eines Faches. Festschrift für Gerhard Helbig zum 65. Geburtstag. München: 389-404.

Burger, Harald (1998): Phraseologie. Eine Einführung am Beispiel des Deutschen. Berlin.

Canisius, Peter (1998): "Kedves Hallgatóink! Possessivpronomina im Deutschen und Possessivsuffixe im Ungarischen". In: Canisius, Peter/Rössler, Paul (1998): Studien zur Germanistik. Fünfter Jahrgang. Pécs: 9-42.

Coseriu, Eugenio (1967): "Lexikalische Solidaritäten". Poetica 1: 293-303.

Deutsches Universalwörterbuch (2003). Ed. vom Dudenverlag. CD-ROM-Ausgabe. Mannheim etc.

Edmondson, Willis/House, Juliane (2000): Einführung in die Sprachlehrforschung. Tübingen.

Helbig, Gerhard/Schenkel, Wolfgang $\left(1991^{8}\right)$ : Wörterbuch zur Valenz und Distribution deutscher Verben. Tübingen.

Hufeisen, Britta/Neuner, Gerhard (2000): Angewandte Linguistik für den fremdsprachlichen Deutschunterricht. Berlin. (= Fernstudieneinheit 16).

Gerner, Zsuzsanna (2001): "Zur Identitätsbildung ungarndeutscher Jugendlicher. Eine Fallstudie". In: Landesselbstverwaltung der Ungarndeutschen (eds.): Deutschunterricht der Ungarndeutschen um die Jahrtausendwende. Sprache und Identitätsbildung. Wissenschaftliche Tagung vom 7.-8. Januar 2000. Budapest: 74-95.

Katsikas, Sergios (2002): Warum machen wir Fehler, wenn wir Fremdsprachen lernen? Ebenen ätiologischer Fehleranalyse und Konsequenzen für die Fehlerbewertung. Unpubliziertes Manuskript eines Vortrags, gehalten am FASK Germersheim am 20.6.2002.

Lakoff, George/Johnson, Mark (1980): Metaphors We Live By. Chicago.

Näf, Anton/Pfander, Doris (2001): " $<$ Springing of $>$ a $<$ bruck $>$ with an elastic $<$ sail $>$. Deutsches im Englisch von französischsprachigen Schülern". Zeitschrift für Angewandte Linguistik 35: 5-39.

Näf, Anton (2001): "Sprachlicher Kontrastmangel als Fehlerquelle". In: Canisius, Peter/Gerner, Zsuzsanna/Glauninger, Manfred Michael (eds.) (2001): Sprache - Kultur Identität. Festschrift für Katharina Wild zum 60. Geburtstag. Studien zur Germanistik. Siebter Jahrgang. Pécs: 231-254.

Reder, Anna (2002): "Eine Aufgaben- und Übungstypologie zur Entwicklung der Kollokationskompetenz von DaF-Lernern". In: Ágel, Vilmos/Herzog, Andreas (eds.): Jahrbuch der ungarischen Germanistik. Budapest/Bonn: 293-315.

Scherfer, Peter (2001): "Zu einigen wesentlichen Merkmalen lexikalischer Kollokationen". In: Lorenz-Bourjot, Martine/Lüger, Heinz-Helmut (eds.): Phraseologie und Phraseodidaktik. Wien: 3-21.

Teubert, Wolfgang (2004): "Feste Wortverbindungen in ein- und mehrsprachiger Sicht". In: Czicza, Dániel/Hegedús, Ildikó/Kappel, Péter/Németh, Attila (eds.): Wertigkeiten, Ge- 
schichten und Kontraste. Festschrift für Péter Bassola zum 60. Geburtstag. Szeged: 521547.

Szû́cs, Tibor (1999): Magyar-német kontrasztiv nyelvészet a hungarológiában. Nemzeti Tankönyvkiadó. Budapest.

Wild, Katharina (1992): "Deutschunterricht und Spracherhalt bei den Ungarndeutschen". In: Spiegel-Schmidt, Friedrich (ed.): Suevia Pannonica. Archiv der Deutschen aus Ungarn. München 10: 5-14. 